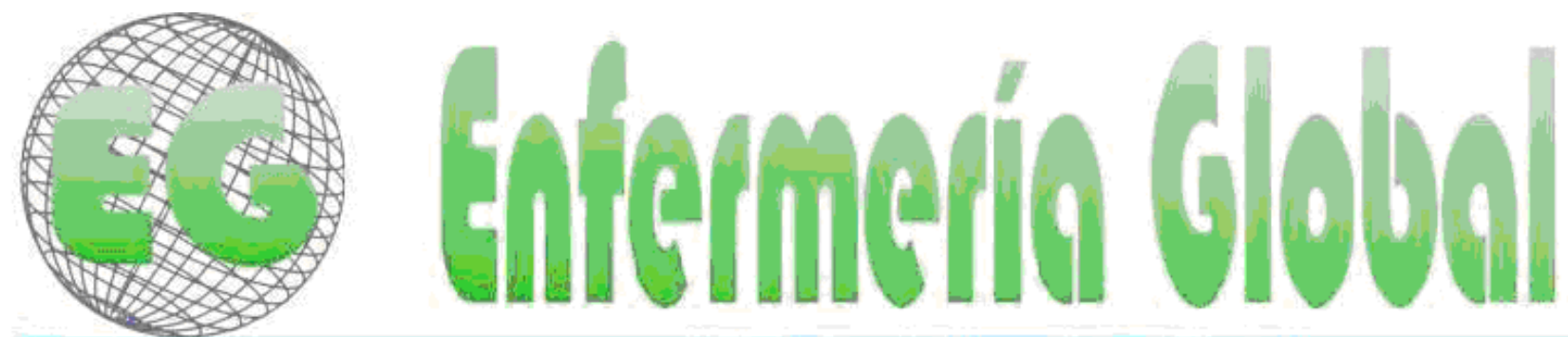

$\mathrm{N}^{\circ} 16$

Revista electrónica cuatrimestral de Enfermeria

Junio 2009

www.um.es/eglobal/

\title{
MISCELANEA
}

\section{LA RECEPTIVIDAD DE LA NOTICIA DE MUERTE ENCEFÁLICA EN LOS FAMILIARES DE DONANTES DE ÓRGANOS Y TEJIDOS PARA TRASPLANTE}

A RECEPTIVIDADE DA NOTÍCIA DA MORTE ENCEFÁLICA NOS FAMILIARES DE DOADORES DE ÓRGÃOS E TECIDOS PARA TRANSPLANTE

\section{"Moreira Cinque, V., " ${ }^{*}$ Ferraz Bianchi, ER.}

\begin{abstract}
*Enfermeiro do Hospital das Clínicas. Especialista em Terapia Intensiva e Mestre pela Escola de Enfermagem da USP. **Enfermeira. Professora Associada da Escola de Enfermagem da USP. São Paulo. Brasil.
\end{abstract}

(Extraído de la disertación " Fatores de stress vivenciados pelos familiares no processo de doação de órgãos e tecidos para transplante" , Escola de Enfermagem da USP, 2008.)

Palabras clave: Trasplante de Órganos, Familia, Muerte Encefálica.

Palavras-chave: Transplante de Órgãos, Família, Morte Encefálica

\section{RESUMEN}

El estudio propone caracterizar la receptividad de la noticia de muerte encefálica (ME) en los familiares que participaron del proceso de la donación de órganos así como verificar el nivel de asociación con las variables de interés. La muestra estuvo constituida por 16 familiares que realizaron la donación en una Organización de Procura de Órganos en la ciudad de São Paulo Brasil en 2007. Las entrevistas fueron efectuadas de marzo a junio de 2008, abarcando la siguiente pregunta: “¿cómo fue el recibir la noticia de la muerte encefálica?" La mayoría de los familiares $62,50 \%$ recibió la noticia de ME intranquila. Por el coeficiente phi, hubo asociación de baja a moderada y significante entre la receptividad de la noticia de la ME y las variables: sexo del familiar, edad del familiar, grado de parentesco y la edad del donante. La posibilidad de recibir la noticia de muerte encefálica tranquila fue 1,6 veces más probable en los parientes en primer grado en la línea recta en relación a los demás. (Odds Ratio $=1,567, p=0,026)$. En conclusión, la comunicación debe ser clara haciendo uso de términos comprensibles, pues el uso de un lenguaje técnico puede dificultar la comprensión de la noticia de ME.

\section{RESUMO}

O estudo propõe caracterizar a receptividade da notícia da morte encefálica (ME) nos familiares que vivenciaram o processo de doação de órgãos e verificar o grau de associação com as variáveis de interesse. A amostra foi constituída de 16 familiares que realizaram a doação em uma Organização de Procura de Órgãos da cidade de São Paulo no ano de 2007. As entrevistas foram realizadas de 
março a junho de 2008, com a seguinte questão norteadora: "Como foi receber a notícia da morte encefálica?" A maior parte, $62,50 \%$ dos familiares recebeu a notícia da ME de forma intranqüila. Pelo coeficiente phi, houve associação de fraca à moderada e significante entre a receptividade da notícia da ME e as variáveis: sexo do familiar, idade do familiar, grau de parentesco e idade do doador. A possibilidade de receber a notícia da ME de forma tranqüila foi 1,6 vezes mais provável nos parentes de primeiro grau na linha reta em relação aos demais (Odds Ratio=1,567, $p=0,026$ ). Concluindo, a comunicação deve ser realizada de maneira clara e com a utilização de termos compreensíveis, pois o emprego de expressões técnicas pode atrapalhar a compreensão da ME.

\section{ABSTRACT}

This study wants to characterize the receptivity of brain death (BD) notification by the family, in regards to the organ donation process and to verify the association level of the interest variables. The sample consisted of 16 families which had donated organs to the Organ Procurement Organization in the city of São Paulo, Brazil in 2007. The interviews were conducted from March to June of 2008, using the following guiding question: "How did you receive the notification of brain death?" The majority, $62.5 \%$ of the families had received this BD notification in an inappropriate manner. According to the phi coefficient, a weak to moderate association was verified along with significant findings between the received BD notification and the variables: gender of the family member, age of the family member, degree of kinship and age of the donor. The possibility of receiving the BD information in an appropriate manner was 1.6 more likely with first-degree relatives of direct lineage, in relation to others (Odds Ratio=1.567, $p=0.026$ ). In conclusion, this communication should be performed in as simple a manner as possible while using clear and understandable terms, as the use of technical terms may hinder the understanding of Brain Death.

\section{INTRODUCCIÓN}

El proceso de donación de órganos y tejidos para transplante es complejo y compuesto por diferentes etapas $^{(1)}$. Se inicia con la identificación de un paciente con muerte encefálica (ME) y finaliza a la conclusión del transplante. ${ }^{(1-2)}$.

Una etapa importante en el proceso de donación es la constatación de la ME. La evolución del concepto y los criterios adoptados para el diagnóstico surgieron en 1959, cuando dos neurólogos franceses, Mollarette e Goulon relataron el coma dépassé, significando un estado más allá del coma ${ }^{(3)}$.

El concepto formal fue propuesto por primera vez en 1968, con una comunicación especial al Journal of the Americam Medial Association, realizado por el comité de la Ad Hoc Harvard Medical School que públicamente redefinió la muerte como "muerte encefálica", con el título de "A Definition of Irreversible Coma"(4).

La ME es establecida por la pérdida definitiva e irreversible de las funciones del encéfalo, teniendo una causa conocida, comprobada y capaz de provocar el cuadro clínico. Los criterios se caracterizan por medio de la realización de exámenes clínicos y complementarios con intervalos de tiempo variables de acuerdo con los determinados grupos etarios $^{(5)}$.

La percepción de la gravedad y la eminente muerte son situaciones traumáticas para la familia, al no sentirse preparada para aceptar la pérdida del ser querido, lo que ocasiona tristeza, desesperación y estrés ${ }^{(6-7)}$. La familia se ve frente a la noticia de que el estado de salud del paciente es muy grave, o en los casos de enfermedad preexistente, es informada de que el cuadro clínico se agravó( ${ }^{7}$. 
Después de la sospecha de la $\mathrm{ME}$, el médico responsable del paciente deberá informar a los familiares $^{(8-9)}$. En esta ocasión la familia del potencial donante tiene contacto con el diagnóstico de $\mathrm{ME}$, del cual, la mayoría de las veces, no tiene conocimiento o no conprende ${ }^{(6)}$.

\section{OBJETIVOS}

Con este estudio se busca caracterizar la receptividad de la noticia de ME en los familiares que vivenciaron el proceso de donación de órganos y tejidos para transplante, así como verificar el grado de asociación con las variables de interés.

\section{METODOLOGÍA}

Se trata de un estudio descriptivo, exploratorio y de campo, con abordaje cuantitativo.

\section{Muestra}

La muestra fue contituida por 16 familiares de donantes de órganos fallecidos que vivenciaron el proceso de donación de órganos en una Organización de Búsqueda de Órganos en la ciudad de São Paulo - Brasil en 2007. Esta muestra alcanzó el poder estadístico de $71 \%$.

\section{Colecta y análisis de los datos}

Las entrevistas fueron realizadas de marzo a junio de 2008, después de la autorización de la institución, de la aprobación del Comité de Ética en Investigación (CEI) y de la firma de la Declaración de Consentimiento Libre y Claro de los participantes. La colecta de datos fue guiada por la siguiente pregunta ¿Cómo fue el recibir la noticia de muerte encefálica? así como un cuestionario estructurado, con las características sócio-demográficas de los familiares y los perfiles demográficos y epidemiológicos de los donantes fallecidos.

El periodo e intensidad del sufrimiento de los familiares que conviven con la pérdida de un ser querido es variable, sin embargo, la fase aguda ocurre entre los dos primeros meses después del fallecimento ${ }^{(7,10)}$. Como precaución, los familiares fueron entrevistados como mínimo tres meses después del fallecimiento del donante.

Se agendaron las entrevistas de acuerdo con el lugar, día y horario determinado por los familiares. Se tomaron los cuidados necesarios para que no coincidiesen con fechas especiales, tales como: fecha de cumpleaños del fallecido, fecha del óbito, fecha de la internación, Dia de los niños, Día de los Padres y otras fechas significativas, a fin de evitar más sufrimiento a los participantes.

Los datos fueron explorados y analizados por medio de la lectura y relectura. Se estableció la codificación numérica, la determinación de la frecuencia con que cada evento se presentó y la agrupación de los items por afinidad.

Para la evaluación del nivel de asociación se utilizó el coeficiente phi $(\Phi)$ para la evaluación del grado de asociaciones, que varía de 0 a 1; cuanto más próximo de 1, mayor es la fuerza de asociación. Se calculó el Odds Ratio (OR) con intervalo de confianza de $95 \%$. El nivel de significancia adoptado fue de $5 \%$. Los análisis estadísticos con $p<0,05$ fueron considerados significantes. 


\section{RESULTADOS}

Los 16 familiares entrevistados tenían de media 41,50 años ( $\pm 10,95)$, valor mínimo de 25 y máximo de 64 años. La mayor frecuencia era de mujeres con 62,50\%. La mayoría, 37,50\% era de religión católica, el tiempo medio de pérdida de los familiares fue de 10,75 meses $( \pm 3,52)$ con una variación de 03,97 a 15,73 meses.

En lo referente al grado de parentesco, $75 \%$ de los parientes fueron de primer grado en línea recta, en esta categoria se incluyeron los padres con $31,25 \%$ y los hijos con $43,75 \%$. En relación a la formación escolar no hubo diferencia significativa entre los niveles primaria, secundaria y superior.

Al caracterizar el perfil sócio-demográfico y epidemiológico de los donantes fallecidos, se encontró que el Accidente Vascular Encefálico (AVE) fue la principal causa de ME con $62,50 \%$ y, en segundo lugar el Traumatismo Craneo-Encefálico (TCE) con 37,50\%. En relación al sexo, hubo discreta diferencia entre el porcentaje de hombres y mujeres con $43,75 \%$ y $56,25 \%$ respectivamente. La edad varió de 15 a 72 años, con una media de 44,44 años $( \pm 18,15)$.

\section{La Receptividad de la Notícia de la ME}

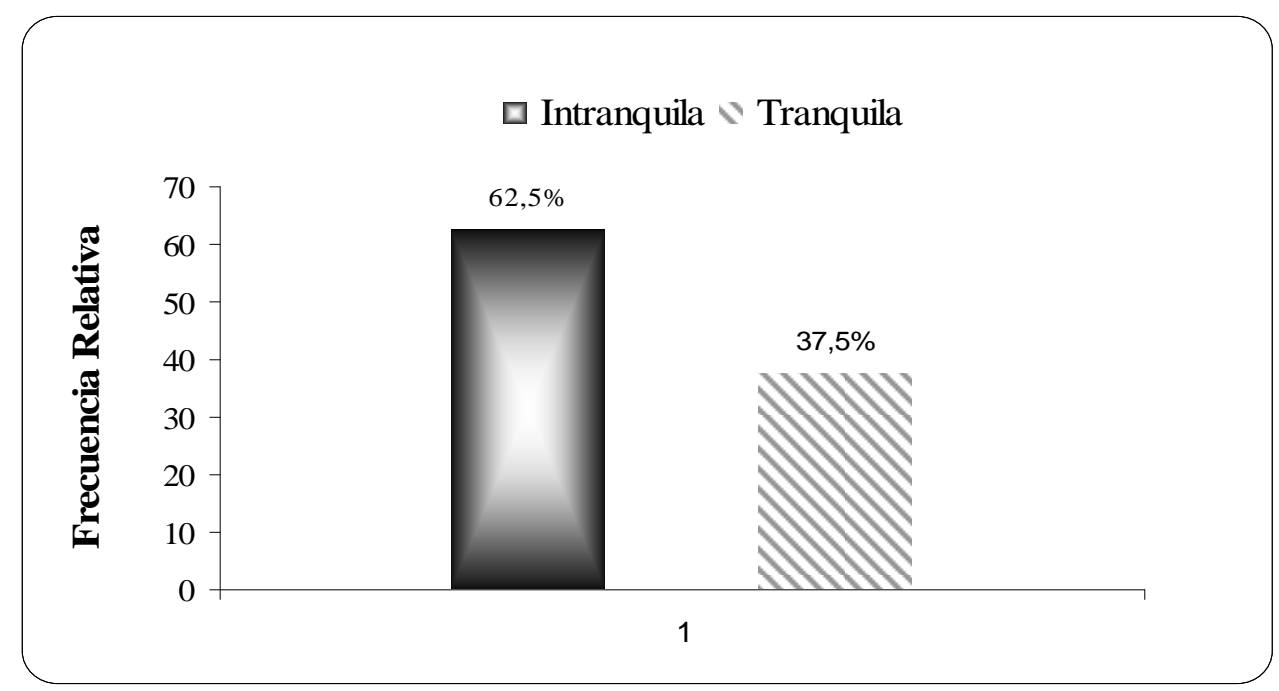

Figura 01 - Distribución de los familiares de los donantes fallecidos, según la receptividad de la noticia de ME, São Paulo - 2008

Innumerables sentimentos y reacciones surgen después de la información de ME a la familia. En esta investigación, se constató que el $62,50 \%$ de los familiares (Figura 01 ) recibieron la noticia de ME de forma intranqüila; el 37,50\% la obtuvo de forma tranquila, de acuerdo con las siguientes declaraciones:

a) "[...] recibí la notícia tranquila porque ya estaba desconfiada y preparada." (Familiar 01)

b) "Como si fuese un shock, todo aconteció [...]." (Familiar 02)

c) "No fue facil, incluso sabiendo que en una semana no tuvo mejora, esperaba una buena noticia [...]." (Familiar 04)

d) "No fue sorpresa, ya habia recibido por parte de la médica de nuestra familia que fue clara que no había alternativa." (Familiar 06) 
e) "Tuvimos la noticia de la manera más cruel que se pudiese, me dijeron así: 'No sé si usted ya sabe, pero ya hicimos todos los exámenes y ya esta confirmada la muerte encefálica y lo que ustedes van a decidir o que es lo que quieren', fue un shock, había conversado con el neurólogo y él me habia dado esperanzas, pero el cuadro era grave y de repente recibimos la noticia." (Familiar 09)

f) "El impacto de la noticia, nadie está preparado para eso, llamaron a casa e informaron que dio flujo y mi hermana no entendió, a pesar de que todo fue muy claro, se quedo descontrolada, diciendo que no iba a la donación que usted (la madre) va a matar a su hijo [...] cualquier cosa hace a la gente tener esperanza." (Familiar 10)

\begin{tabular}{|c|c|c|c|c|c|}
\hline Variables & $\begin{array}{l}\text { Odds } \\
\text { Ratio }\end{array}$ & $\begin{array}{l}\text { Intérvalo de } \\
95 \%\end{array}$ & Confianza & $\Phi$ & $\begin{array}{l}p \\
\text { valor }\end{array}$ \\
\hline Sexo del familiar & 0,689 & $0,498-0,954$ & & $\begin{array}{l}- \\
0,187\end{array}$ & $0,024^{*}$ \\
\hline Edad del familiar & 0,186 & $0,131-0,265$ & & 0,377 & $<0,001^{*}$ \\
\hline $\begin{array}{ll}\text { Grado } & \text { de } \\
\text { parentesco } & \end{array}$ & 1,567 & $1,053-2,330$ & & 0,186 & $0,026^{*}$ \\
\hline Edad del donador & 0,448 & $0,326-0,615$ & & $\begin{array}{l}- \\
0,194\end{array}$ & $<0,001^{*}$ \\
\hline
\end{tabular}

$$
{ }^{*} p<0,05
$$

El valor de phi (Tabla 01) apuntó asociación de débil a moderada, sin embargo, positivo y significativo entre la receptividad de la noticia de ME y el grado de parentesco. Se verificó no sólo la asociación de débil a moderada, sino también de forma inversa y significativo entre la receptividad de la noticia de ME y las variables: sexo del familiar, edad del familiar y edad del donador.

En este caso, la posibilidad de recibir la noticia de ME de forma tranquila fue menos probable en los familiares: de sexo femenino $(\mathrm{OR}=0,689)$, con menos de 40 años $(\mathrm{OR}=0,186)$, así como los familiares cuyo donante tiene menos de 40 años $(\mathrm{OR}=0,448)$. Sin embargo, los parientes de primer grado en linea directa, fueron 1,6 vezes más propensos a recibir la noticia de ME de forma tranquila en relación a los demás familiares.

\section{DISCUSIÓN}

Informar a los familiares, sobre la gravedad del cuadro clínico del paciente y el inicio de los exámenes para verificar la ME es un elemento fundamental, promoviendo de esta forma la preparación de la familia para el inicio del proceso de luto, además de realizar el proceso de donación de órganos de forma transparente, respaldado en los princípios éticos y legales.

Para la familia, la muerte no representa solo un proceso biológico, también un proceso emocional y cognitivo, considerado bastante perturbador ${ }^{(7)}$.

En los Estados Unidos, al analizar los factores relacionados a la comprensión de la ME de 403 familias, se constató que $21 \%$ de los entrevistados tenían esperanza en la recuperación del cuadro clínico, dentro de ellos el grupo etário más elevado. Las personas que entendieron y aceptaron la ME fueron más propensas a donar ${ }^{(11)}$. 
La incomprensión del la ME dificulta la toma de decisión en relación a la donación ${ }^{(6,12-13)}$. En la literatura fueron encontrados resultados en que el desconocimiento y la no aceptación de la ME representaron $83 \%(n=25)$ de los motivos para negarse a la donación ${ }^{(14)}$.

En esta investigación, se verificó que $62,50 \%$ de los familiares recibieron la noticia de la ME de forma intranquila, al tiempo que $37,50 \%$ la obtuvieron de forma tranquila. El coeficiente $\Phi$ apuntó asociación de débil a moderada y significante entre la receptividad de la noticia de la ME y las variables: sexo del familiar, edad del familiar, grado de parentesco, y edad del donador. La posibilidad de recibir la noticia de la ME de forma tranquila fue menos probable en los familiares del sexo femenino $(\mathrm{OR}=0,689)$. Sin embargo, los parientes de primer grado en linea directa, fueron 1,6 veces más propensos a recibir la noticia de la ME de forma tranquila en relación a los demás familiares.

La familia se queda confusa al recibir la información de la ME sin esclarecimientos previos sobre la posibilidad de ocurrencia de esta situación ${ }^{(15)}$. La falta de conocimiento, por parte de las familias, se refleja en la inseguridad que acaba en el estrés, por permitir la donación y pensar que la otra persona puede estar viva, dificultando, al principio, la toma de decisión y, después, la convivencia con la decisión de haber donado ${ }^{(16)}$.

La transparencia del proceso de donación es un factor esencial, en el cual la familia debe ser debidamente informada y esclarecida sobre el cuadro del ser querido, pues la falta de claridad es percibida como una condición que genera duda, angustia, dolor y desesperación ${ }^{(6,7,13,15-16)}$. Son necesarias informaciones sobre la ME, disminuyendo sus dudas en relación al tiempo transcurrido para culminar los exámenes que comprueben la ME. En esta ocasión los familiares se encuentran frágiles emocionalmente para responder a las preguntas sobre la donación ${ }^{(17)}$.

En este sentido, la Resolución n. 292/2004 del Consejo Federal de Enfermería (Brasil) determina que compete al enfermero planear, ejecutar, cordinar, supervisionar y evaluar los procedimientos de enfermería prestados a los familiares y donantes de órganos y tejidos. Durante la entrevista con la familia y representante legal, deben fortalecerse las informaciones sobre el proceso de donación, incluyendo la información clara sobre el diagnóstico de la ME.

La noticia de la ME es evaluada por la familia como una situación estresante, sea por la falta de claridad necesaria sobre el estado del paciente o hasta por sentir dudas en relación al diagnóstico de la ME. La falta de información, el hecho del cuerpo estar caliente y el corazón aún estar palpitando, dificulta la comprensión de la ME, pudiendo indicar que la persona pueda estar viva incluso con las pruebas presentadas.

Se espera que los datos de la presente investigación lleven a los enfermeros, sociedades y cursos de graduación a reflexionar y considerar la noticia de la ME compleja y estresante para la familia. La comunicación debe ser realizada de forma clara y con el uso de términos comprensibles, pues el empleo de un lenguaje técnico puede dificultar la comprensión de la ME.

\section{CONCLUSIONES}

Los resultados de esta investigación permitieron concluir que:

1. La mayoría de los familiares recibió la noticia de la ME de forma intranquila $(62,50 \%)$. 
2. La asociación fue de débil a moderada y significativa entre la receptividad de la noticia de la ME y variables: sexo del familiar, edad del familiar, grado de parentesco y edad del donante.

- La posibilidad de recibir la noticia de la ME de forma tranquila fue menos probable en los familiares: del sexo femenino $(\mathrm{OR}=0,689)$, con menos de 40 años $(\mathrm{OR}=0,186)$, así como en los familiares cuyo donante tenía menos de 40 años $(\mathrm{OR}=0,448)$.

- Los parientes de primer grado en linea recta fueron 1,6 veces más propensos a recibir la noticia de la ME de forma tranquila en relación a los demás familiares.

\section{REFERENCIAS}

1. São Paulo (Estado). Secretaria de Estado da Saúde de São Paulo. Coordenação do Sistema Estadual de Transplante. Doação de órgãos e tecidos. São Paulo; 2002.

2. Pereira WA, Fernandes RC, Soler WV. I Reunião de Diretrizes para Captação e Retirada de Múltiplos Órgãos. São Paulo: Associação Brasileira de Transplante de Órgãos; 2003.

3. Mollaret P, Goulon M. Le coma dépassé. Rev Neurol (Paris). 1959;101:3-15.

4. A definition of irreversible coma. Report of the Ad Hoc Committee of the Harvard Medical School to examine the definition of brain death. JAMA. 1968;205(6):337-40.

5. Conselho Federal de Medicina (CFM). Resolução CFM n.⒈480, de 8 de agosto de 1997. Critérios para a caracterização de morte encefálica. Diário Oficial da União, Brasília, 21 ago. de 1997. Seção 1, p. 18.227-8.

6. Moraes EL. A recusa familiar no processo de doação de órgãos e tecidos para transplante [dissertação]. São Paulo: Escola de Enfermagem, Universidade de São Paulo; 2007.

7. Bousso RS. Um tempo para chorar: a família dando sentido à morte prematura do filho [tese livre-docência]. São Paulo: Escola de Enfermagem, Universidade de São Paulo; 2006.

8. Brasil. Lei n. ${ }^{\circ} 9.434$, de 4 de fevereiro de 1997 . Dispõe sobre a remoção de órgãos, tecidos e partes do corpo humano para fins de transplante, e dá outras providências. Diário Oficial da União, Brasília, 5 de fev. de 1997. p. 2191-3.

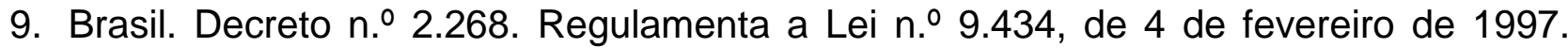
Dispõe sobre a remoção de órgãos, tecidos e partes do corpo humano para fins de transplante e tratamento, e dá outras providências. Diário Oficial da União, Brasília, 1ํㅡㄴ de jul. de 1997. p. 13739 - 42.

10. Maciejewski PK, Zhang B, Block SD, Prigerson HG. An empirical examination of the stage theory of grief. JAMA. 2007;297(7):716-23.

11. Siminoff LA, Mercer MB, Arnold R. Families' understanding of brain death. Prog Transplant. 2003;13(3):218-24. 
12. Sque M, Long T, Payne S. Organ donation: key factors influencing families' decisionmaking. Transplant Proc. 2005;37(2):543-6.

13. Ripoll Espiau F. Donación-trasplante de órganos concienciación de los profesionales sanitarios y población: sensibilización a través de los médios. Enfermería Global [periódico na Internet]. 2003 maio [citado 2008 set. 05];(2):1-13. Disponível em: http://revistas.um.es/eglobal/article/viewFile/663/695

14. Singh $P$, Kumar A, Sharma RK. Factors influencing refusal by relatives of brain-dead patients to give consent for organ donation: experience at a transplant centre. J Indian Med Assoc. 2004;102(11):630,632, 643.

15. Santos MJ, Massarollo MCKB. Processo de doação de órgãos: percepção de familiares de doadores cadáveres. Rev Latino-am Enfermagem. 2005;13(3):382-7.

16. Sadala MLA. A experiência de doar órgãos na visão de familiares de doadores. J Bras Nefrol. $2001 ; 23(3): 143-51$.

17. Long $\mathrm{T}$, Sque $\mathrm{M}$, Payne $\mathrm{S}$. Information sharing: its impact on donor and nondonor families' experiences in the hospital. Prog Transplant. 2006;16(2):144-9. 